\title{
Effects of humidity on the electrical properties of thermal inkjet-printed films of copper tetrasulfonated phthalocyanine (CuTsPc) onto paper substrates
}

\author{
Tiago Carneiro Gomes • Rafael Furlan de Oliveira • \\ Élder Mantovani Lopes • Maykel dos Santos Klem • \\ Deuber Lincon da Silva Agostini • Carlos José Leopoldo Constantino • \\ Neri Alves
}

Received: 29 October 2014/Accepted: 4 December 2014/Published online: 12 December 2014

(C) Springer Science+Business Media New York 2014

\begin{abstract}
The effects of humidity on the electrical properties of thermal inkjet-printed copper tetrasulfonated phthalocyanine (CuTsPc) films onto paper substrates are reported. DC electrical measurements revealed that sample resistance decreases from $10^{12} \Omega$ in moderate vacuum to approximately $10^{7} \Omega$ at highly water-saturated atmosphere. This behavior is attributed to the creation of water pathways on the cellulose fibers that allow ionic species such as impurities, protons $\left(\mathrm{H}^{+}\right)$, and dissociated $\mathrm{Na}^{+}$ions from the CuTsPc molecules, to flow. The contribution of the CuTsPc molecules and their dissociated $\mathrm{Na}^{+}$ions on the sample resistance was elucidated analyzing the electrical response of bare and printed paper substrates. An increase of relative humidity levels $(\mathrm{RH})$ from $10 \%$ to $40-45 \%$
\end{abstract}

T. C. Gomes · R. F. de Oliveira $(\bowtie) \cdot$ M. dos Santos Klem ·

D. L. da Silva Agostini - C. J. L. Constantino · N. Alves

Faculty of Science and Technology, São Paulo State University -

UNESP, Presidente Prudente, SP 19060-900, Brazil

e-mail: furlanrafa@hotmail.com.br

T. C. Gomes

e-mail: tiagocunesp@gmail.com

M. dos Santos Klem

e-mail: maykel_klem@live.com

D. L. da Silva Agostini

e-mail: deuber@fct.unesp.br

C. J. L. Constantino

e-mail: case@ @ct.unesp.br

N. Alves

e-mail: neri@fct.unesp.br

É. M. Lopes

Federal Institute of Education, Science and Technology of Santa

Catarina, Araquari, SC 89245-000, Brazil

e-mail: elder.mantovani@ifc-araquari.edu.br revealed an increase of current of four orders of magnitude for printed samples, whereas less than one order of magnitude was registered for bare paper substrates. Electrical measurements as a function of temperature have shown that moisture can inhibit the semiconducting properties of CuTsPc. Thermal gravimetric studies revealed that approximately $11 \%$ of mass accounting to the presence of water is released above $70{ }^{\circ} \mathrm{C}$, the same temperature in which the sample recovers its dry-state semiconducting behavior. The semiconducting properties of CuTsPc can also be observed upon white light illumination, whereas the charge carrier extraction is governed by the RH level. These results point out the important role of humidity on the electrical properties of paper-based organic, electronic, and optoelectronic devices.

\section{Introduction}

Metallophthalocyanines (MPcs) are organic macrocyclic semiconductors that have been used in several electronic devices such as light-emitting diodes [1,2], photovoltaic cells [3-7], and field-effect transistors [8-10]. In these applications, MPcs are usually deposited as thin films by thermal evaporation with very precise control since molecular organization plays an important role on the film electrical properties [11, 12]. However, the current trend of organic electronics toward the production of large area and low-cost devices from solution-processable materials using printing techniques might restrict the use of MPcs due to their low solubility in many common organic solvents [1317]. Hence, the use of substituted phthalocyanine derivatives such as water-soluble tetrasulfonated phthalocyanines $(\mathrm{TsPc})$ represents a very attractive alternative for printed electronics. 
Nevertheless, despite the good perspectives for TsPcbased electronics, the number of reports related to this topic is rather limited [6, 18-23], principally involving the production of devices using printing methods [22, 23]. Most of applications exploiting the water solubility of TsPc molecules are based on layer-by-layer (LbL) assembly for sensors [19, 24-26]. LbL has also been used to fabricated TsPc-based field-effect transistors [18] and organic solar cells [19], whereas other deposition strategies include the electrodeposition of copper TsPc-based films for dye-sensitized solar cells [6] and spin coating of nickel, aluminum, cobalt, zinc, and copper TsPcs for organic field-effect transistors [21, 27]. Regarding the publications using printed TsPcs, Lian et al. produced memory devices using copper TsPc and poly(3,4-ehthylenedioxythiophene:polystyrene sulfonate) (PEDOT:PSS) onto plastic substrates [22], whereas Kim et al. reported the preparation of several TsPcs inks for color filters in liquid crystal displays [23].

An interesting and novel strategy for printed electronics relies on the deposition of electronic materials onto paper substrates [28-30]. The use of paper as a platform for device manufacturing, known as paper electronics [28-30], is very attractive due to several properties offered by paper substrates [29]. Paper is by far the cheapest available flexible substrate [29] (paper is around 20 times cheaper than polyethylene terephthalate-PET, for example), it is highly available in variety of forms, mechanically robust, it can be easily cut and folded, and it is also environmentalfriendly $[28,29]$. Depending on the envisioned application other characteristics exhibited by paper as opacity, chemical and thermal stability, weightlessness, and porosity can also be explored. Due to all advantages offered by paper electronics, many reports in literature deal with the fabrication of paper-based organic devices for energy storage [31-33], as transistors [34-38], photodiodes [39], and solar cells [40]. However, just a few articles describe the production of paper-based organic devices using printing technologies [32, 36, 37]. To the best of our knowledge, the fabrication of TsPc-printed devices onto paper substrates is one of the issues on paper electronics that has not been exploited yet.

To fill this gap and to address more information about the feasibility of TsPc-based printed devices, we report the thermal inkjet printing of copper tetrasulfonated phthalocyanine $(\mathrm{CuTsPc})$ onto paper substrates and discuss the effects of humidity on sample electrical properties. The use of printing methods, in specific the thermal inkjet one, is attractive due to the possibility to use desktop printers for real cost-effective device fabrication [41]. Although this method has, as the main limitation, the use of inks with specific properties [41, 42] (viz., viscosity, boiling point, and surface tension), water-soluble CuTsPc molecules have shown to be suitable candidates for the fabrication of electronic devices. Moreover, the well-known compatibility between inkjet desktop printers and paper sheets allows rapid device prototyping at low cost.

In this work, we describe the preparation and printing of a CuTsPc-based ink onto paper substrates and the evaluation of sample electrical characteristics under different humidity conditions. The sample overall electrical response was attributed to ionic and electronic contributions from $\mathrm{CuTsPc}$ molecules and the paper itself. Current-temperature measurements and complementary thermogravimetric (TGA) and differential scanning calorimetric (DSC) analyses revealed the occurrence of two phenomena involving the entrapment of water molecules within the CuTsPc structure, which affects its semiconducting properties. The sample electrical response was analyzed also under illumination and its photoconductive properties are discussed.

\section{Experimental details}

\section{CuTsPc ink preparation and printing}

The inkjet printing of electronic materials using commercial desktop printers demands the use of solutions with properties (viz., surface tension, viscosity, and boiling point) similar to those of standard inks [41]. For this purpose, we prepared CuTsPc aqueous solutions $\left(20 \mathrm{mg} \mathrm{mL}^{-1}\right)$ dissolving copper phthalocyanine- $3,4^{\prime}, 4^{\prime \prime}, 4^{\prime \prime \prime}$-tetrasulfonic acid tetrasodium salt powder, $M_{\mathrm{W}} 984.25 \mathrm{~g} \mathrm{~mol}^{-1}$ and dye content $85 \%$ (Sigma-Aldrich Ltd), in a mixture of water and ethylene glycol with a ratio of 2:1 (v:v). The resulting ink was left in a magnetic stirrer for 30 min followed by sonication during $15 \mathrm{~min}$ to assist its solubilization. Finally, the CuTsPc ink was carefully filtered using a PTFE syringe filter (pore size of $0.45 \mu \mathrm{m}$ ).

Before receiving the CuTsPc ink, the printer cartridge (Hewlett-Packard number 21) was opened and the original ink, internal sponge, and filter were removed. The cartridge was cleaned with n-methyl-2-pyrrolidone (NMP), isopropyl alcohol, and deionized water $(1: 1: 1 \mathrm{v}: \mathrm{v}: \mathrm{v})$ and left in ultrasonic bath for $20 \mathrm{~min}$ to remove all residues. The samples were designed as $10 \times 25 \mathrm{~mm}$ strips using Microsoft Word ${ }^{\circledR}$ software. The printing parameters were set to RGB $(0,0,0)$, at the lowest speed and maximum printing quality, which means the largest number of pixels per inch that can be achieved by the printer. Successive printings (1-10 prints) of CuTsPc were carried out using a commercial Hewlett-Packard Deskjet 2460 printer onto bond paper substrates at a fixed position. Lastly, gold electrodes ( $70 \mathrm{~nm}$-thick) were thermally evaporated at the sample edges, $3 \mathrm{~mm}$ spaced each other. The final sample architecture and the chemical structure of $\mathrm{CuTsPc}$ are depicted in Fig. 1. 
Fig. 1 Inkjet-printed CuTsPc on paper substrates: sample dimensions and $\mathrm{CuTsPc}$ molecular structure

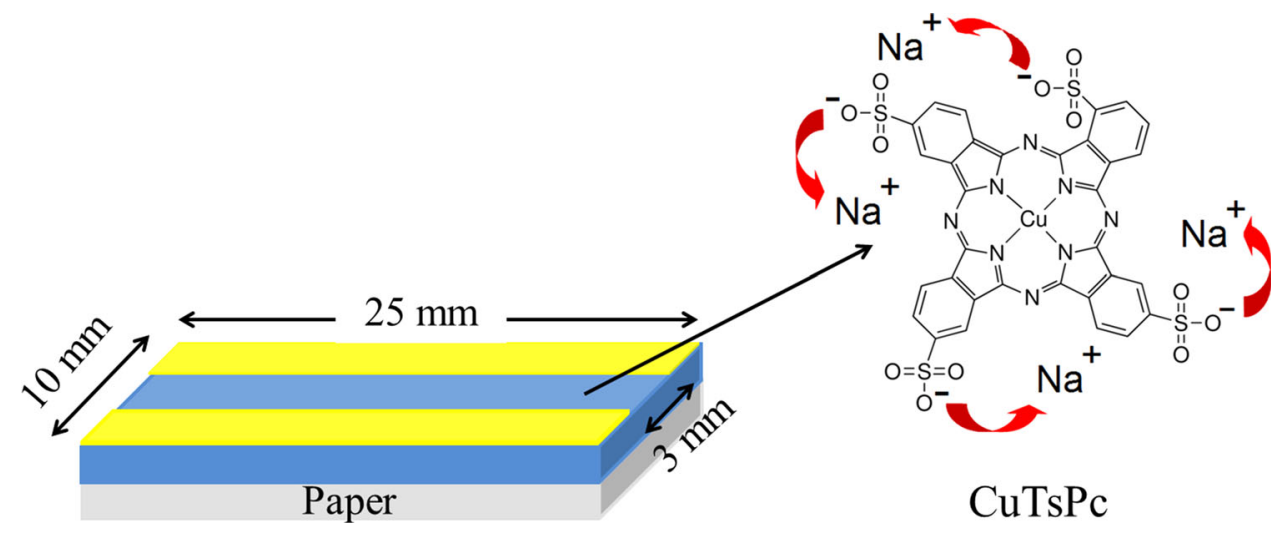

Electrical and thermal characterization of printed CuTsPc films

The influence of different relative humidity $(\mathrm{RH})$ levels on the electrical properties of thermal inkjet-printed CuTsPc films onto paper was investigated by means of currentvoltage $(\mathrm{I}-\mathrm{V})$ and current-time (I-t) measurements in dark and upon white light exposure. Electrical measurements were carried out using a Keithley 617 source meter unit in a home-made sample holder under moderate vacuum ( $\sim 10^{-1}$ Torr), with RH at approximately $10 \%, 40-45 \%$, and at high moisture content. Low RH levels $(\sim 10 \%)$ were achieved adding silica gel beads into the sample holder, whereas high RH level was obtained using an opened water-filled vial in the sample holder. Measurements at medium RH levels (40-45\%) were performed exposing the sample to the laboratory atmosphere and monitoring the room humidity using a thermo hygrometer. Prior to the electrical characterization in vacuum and $\mathrm{RH}$ $\sim 10 \%$, the sample was left overnight in the presence of silica for drying. For other RH values the waiting time was approximately $4-5 \mathrm{~h}$.

To elucidate the humidity effects on the electrical properties of CuTsPc films, thermogravimetry (TGA) and differential scanning calorimetry (DSC) analyses were performed. The TGA was carried out with a TGA Netzsch equipment (model 209), heating the CuTsPc powder $(5 \mathrm{mg})$ in alumina crucible using purified nitrogen gas $\left(15 \mathrm{~mL} \mathrm{~min}{ }^{-1}\right.$ ) at a heating rate of $10^{\circ} \mathrm{C} \mathrm{min}^{-1}$ from 27 to $700{ }^{\circ} \mathrm{C}$. The DSC measurements were carried out using a Netzsch, DSC 204-Phoenix in alumina crucible with lid pierced at the center. The heating rate $10{ }^{\circ} \mathrm{C} \min ^{-1}$ was employed over a temperature range of $-140{ }^{\circ} \mathrm{C}$ at $500{ }^{\circ} \mathrm{C}$ in inert $\mathrm{N}_{2}$ atmosphere with a flow of $25 \mathrm{~mL} \mathrm{~min}^{-1}$.

Current-time measurements upon light exposure were performed using a commercial fluorescent lamp bulb (white light $23 \mathrm{~W}$ ) interspaced from sample by a $2 \mathrm{~cm}$ thick transparent acrylic window to avoid thermal effects from the bulb. I-t currents were registered during $15 \mathrm{~min}$ of illumination applying $0.5 \mathrm{~V} \mathrm{DC}$ bias at RH approximately $10 \%$ and $40-45 \%$.

\section{Results and discussion}

The humidity effects on the electrical properties of inkjetprinted $\mathrm{CuTsPc}$ onto paper were initially investigated as a function of the number of prints. To that $\mathrm{I}-\mathrm{V}$ measurements (in dark) were performed sweeping DC bias from -1.0 to $+1.0 \mathrm{~V}$ for samples containing from 1 to 10 prints of CuTsPc over the same position on the paper substrate. The sample resistance was calculated from Ohm's law and the result is shown in Fig. 2.

From Fig. 2a one can observe that resistance decreases progressively from $10^{12} \Omega$ (in vacuum) to $10^{7} \Omega$, independently of the number of prints, as the $\mathrm{RH}$ levels increase up to water-saturated atmosphere. This behavior is consistent with the exponential decay of cellulose resistivity as a function of RH reported by Murphy [43]. As water molecules adsorbs to hydroxyl groups of the cellulose fibers, a water pathway is created and $\mathrm{H}^{+}$ions and ionic species from impurities can flow [29]. Though, the charge transport within paper is not completely understood and comparisons about their electrical characteristics must be careful because cellulose-based materials are very different in composition and can also present anisotropic properties [29]. Figure $2 \mathrm{a}$ also shows rather constant resistances for different number of prints. A detailed inspection in the obtained values (for $\mathrm{RH} \sim 45 \%$ ) revealed a decay in resistance for the first five prints followed by a constant trend as the number of prints further increases, as illustrated in Fig. 2b. This effect occurs for all tested conditions (result not shown). The inkjet printing of CuTsPc covers progressively the paper fibers causing a decrease in the sample electrical resistance due to the semiconducting properties of phthalocyanine molecules $[44,45]$ but also to the addition of water and dissociated $\mathrm{Na}^{+}$ions from the CuTsPc ink into the paper. The constant 

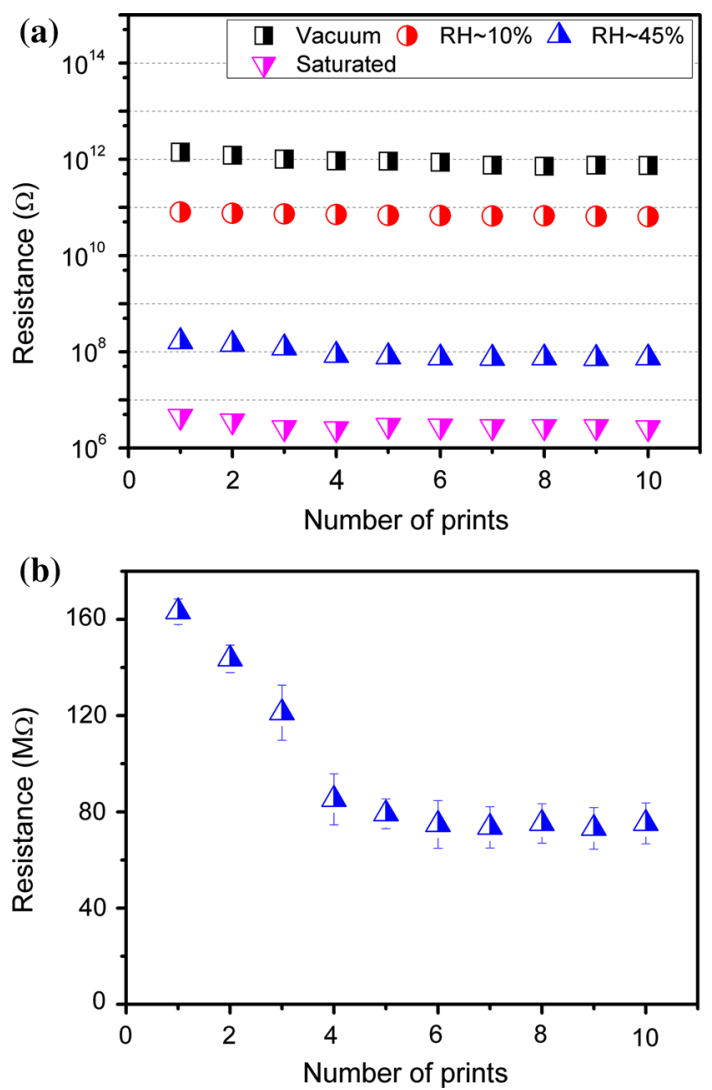

Fig. 2 Electrical resistance of CuTsPc-printed samples $\mathbf{a}$ as a function of the number of prints for different humidity levels and b as a function of the number of prints for $\mathrm{RH} \sim 40-45 \%$

behavior of the resistance values after the fifth print suggests that each printing step further increases the fibers coverage and make the ink penetrates deeper into the paper volume. After the fifth print the fiber capability to absorb ink reaches a limit and ink uptake only occurs within deeper regions in the paper that do not contribute to the sample overall electrical response.

To distinguish the contributions from paper and $\mathrm{CuTsPc}$ on the sample electrical response in the presence of moisture, we performed current-time measurements (in dark) on CuTsPc-covered (5 prints) and uncovered paper. Measurements were carried out applying $0.5 \mathrm{~V}$ for $10,000 \mathrm{~s}$ while exposing the sample to a rapid increase of humidity (from approximately $10 \%$ to $40-45 \%$ ). These results are shown in Fig. 3.

At RH approximately $10 \%$, CuTsPc-covered and uncovered paper exhibited current values of $10^{-11} \mathrm{~A}$ in agreement with results shown in Fig. 2. The sudden raise of RH to $\sim 40-45 \%$ provokes an increase of current in both samples. For bare paper the current change is rather small (less than 1 order of magnitude), whereas CuTsPc-covered paper showed current variations of 4 orders of magnitude. The response time $(\tau)$ of CuTsPc-covered and uncovered

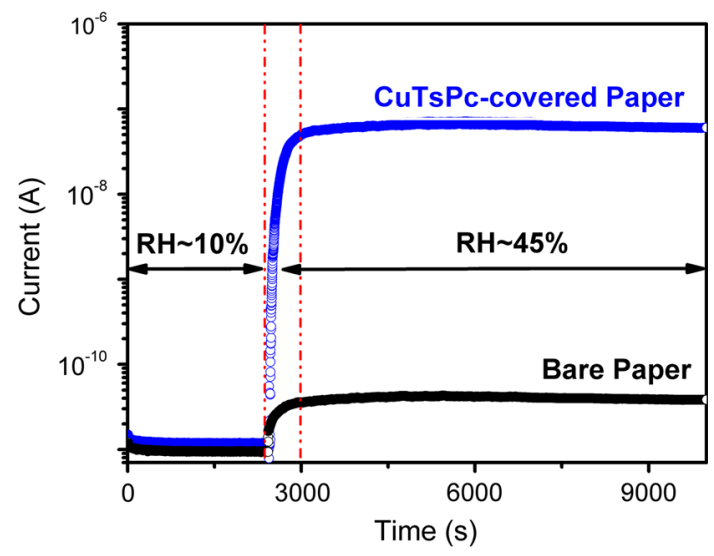

Fig. 3 Current-time response (in dark) of CuTsPc-covered (5 prints) and uncovered paper upon RH change from 10 to $45 \%$

paper toward moisture absorption was estimated fitting a first-order exponential curve (data not shown), whereas $\tau=400$ and $480 \mathrm{~s}$, respectively. The similarity between $\tau$ values suggests that water uptake is dominated mainly by the paper, as expected. The slightly smaller values of $\tau$ for CuTsPc-printed paper can be related to the swelling of fibers caused by the printing procedure, which can make moisture flowing faster inwards the paper [29]. The most relevant information from Fig. 3 is that humidity interacts with $\mathrm{CuTsPc}$ and plays some role on its electrical conductivity. The electronic conductivity of MPcs is known to be dependent on the presence of atmospherical chemical species, mainly oxidizing and reducing gases [46-49]. Molecular oxygen $\left(\mathrm{O}_{2}\right)$, for instance, has been assigned to promote coordinative interactions with phthalocyanine metal centers, which withdraw its electrons to generate positive charge carries (holes) in the material bulk [50]. Otherwise MPcs are insulating in high vacuum and dark environments [46, 51]. Regarding humidity effects, Bohrer et al. observed electrical current changes on cobalt and metal-free phthalocyaninebased chemiresistors due to the device exposure to water vapor, whereas Karimov and co-workers registered capacitance changes on copper phthalocyanine-coated electrodes for different $\mathrm{RH}$ values [51, 52]. According to Bohrer et al. vapor molecules in general interact at phthalocyanine free sites but can also compete for $\mathrm{O}_{2}$-bound sites [51, 53-55] and Karimov et al. expect that water molecules can act as p-type dopants in MPcs similar to the $\mathrm{O}_{2}$ effect [52]. Harbeck et al., using quartz crystal microbalance sensors and Fourier transform infrared (FTIR) spectroscopy, observed that water sorption in fact occurs at the core of substituted MPcs as well as weak hydrogen bonds in $\mathrm{N}-\mathrm{H}$ groups of metal-free phthalocyanines [54]. Concerning sulfonatesubstituted MPcs, Jarota et al. identified possible sites of interaction for water in aluminum-chloride TsPc using infrared absorption and Raman spectroscopies [53]. These 
sites include the phthalocyanine metal center, the nitrogen atoms in the macrocycle, and the sulfonyl substituents [53]. Jarota et al. also highlight the importance in understanding the mechanisms of interaction between the water and phthalocyanine macrocycles and its effect on the electronic, conduction, and charge transfer states of MPcs. Therefore, in addition to a possible water doping effect in MPcs, sulfonate-substituted MPcs are usually salts or acids then one can expect a strong dependence of TsPcs conductivity on humidity due to dissociation of ionic groups. Here, as we use CuTsPc, dissociated $\mathrm{Na}^{+}$ions are deposited during material printing and moisture assist their flow across the sample, similarly to the effect described for ionic impurities within bare paper. However, for CuTsPc-printed paper this effect is more pronounced due to the large amount of deposited $\mathrm{Na}^{+}$ ions (4 ions for each CuTsPc molecule).

The humidity effects on the electrical properties of CuTsPc-printed paper substrates were also investigated measuring the sample resistance as a function of temperature, as illustrated in Fig. 4. The measurements were carried out in moderate vacuum, RH approximately $10 \%$ and $40-45 \%$ for temperatures ranging from 30 to $150{ }^{\circ} \mathrm{C}$.

From Fig. 4, one can observe that resistance decreases as the temperature rises from 30 to $150{ }^{\circ} \mathrm{C}$ in vacuum and at $\mathrm{RH} \sim 10 \%$. Here, the typical insulating behavior of MPcs in vacuum is not observed since it requires the presence of high vacuum environments. The electrical response of CuTsPc-printed films on paper is more suitable to the behavior of semiconducting materials in which an increase of temperature promotes charge carriers to the conduction band and thus reduces the sample resistance. Here, the resistance values acquired in vacuum are shifted downwards for measurements taken at RH $\sim 10 \%$ characterized by the same slope for a linear fit (data not shown). This suggests that resistance changes from vacuum to $\mathrm{RH}$ $\sim 10 \%$ are just related to the water content within the sample and not to additional phenomena. For RH



Fig. 4 Electrical resistance of CuTsPc-printed samples (5 prints) as a function of temperature for different $\mathrm{RH}$ levels $\sim 40-45 \%$ resistance initially increases but starts to fall above $70{ }^{\circ} \mathrm{C}$. At this humidity level the electrical current is dominated by ionic transport, the rise of temperature in the range $30-70{ }^{\circ} \mathrm{C}$ possibly disturbs the ionic mobility, increasing slightly the sample resistance. At $70{ }^{\circ} \mathrm{C}$ water starts to be removed from the sample and the dry-state semiconducting behavior of $\mathrm{CuTsPc}$ is recovered. This statement is supported by TGA, its first derivative (DTG) and DSC curves of the CuTsPc powder shown in Fig. 5.

From Fig. 5, TGA/DTG curves revealed a mass loss of approximately $11 \%$ corresponding to the peak at $67{ }^{\circ} \mathrm{C}$ and the shoulder at $132{ }^{\circ} \mathrm{C}$. The DSC curve indicates that an endothermic event is occurring at $93{ }^{\circ} \mathrm{C}$ for such peak and at $131{ }^{\circ} \mathrm{C}$ for the shoulder, which are related to water removal from the CuTsPc. This is the water content that affects the electrical properties of CuTsPc. Additional water molecules (1.1\% of mass) strongly interacting with the $\mathrm{CuTsPc}$ moiety are released up to approximately $400{ }^{\circ} \mathrm{C}$. Beyond this temperature the organic structure of CuTsPc starts to degrade, which corresponds to DTG maximum at $500{ }^{\circ} \mathrm{C}$ [56]. This event extends up to $650{ }^{\circ} \mathrm{C}$ and corresponds to a mass loss of $67 \%$.

The electrical properties of inkjet-printed $\mathrm{CuTsPc}$ samples were also evaluated by current-time measurements upon white light illumination at RH approximately $10 \%$ and $40-45 \%$, as shown in Fig. 6. Prior to the light exposure the sample was held at $0.5 \mathrm{~V}$ to allow the current to reach relatively constant values since truly steady-state currents in paper-based devices can occur in a timescale of several hours [29].

As the sample is illuminated, one can observe an increase of current for both RH levels. The initial current variation provided by the light exposure is similar for the two conditions (current peak equal to $1.45 \%$ of the current values in dark). Afterward, the initial current decays and the sample response behaves differently according to the RH. It is well-known that MPcs and their derivatives show intrinsic photoconductivity and photovoltaic effects $[3,45$,

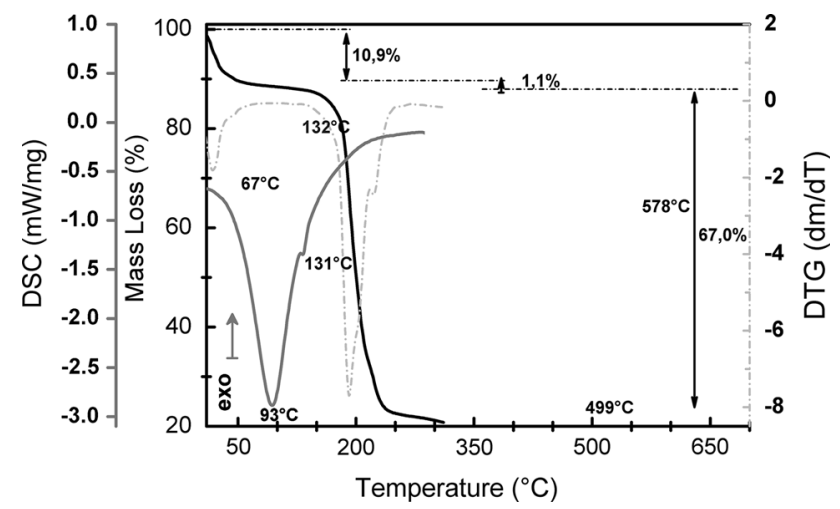

Fig. 5 Thermal analyses of CuTsPc powder. TGA (black), DTG (light gray) and DSC (dark gray) curves 




Fig. 6 Current-time response of CuTsPc-printed samples (5 prints) upon white light exposure for RH 10 and $45 \%$

57]. Here, the sample photocurrent increases continuously at $\mathrm{RH} \sim 10 \%$, whereas a steady-state behavior is reached for RH $45 \%$ (Fig. 6). These two situations can be illustrated as a charging capacitor in which the area under the curve corresponds to the stored charge. Indeed, Karimov et al. observed capacitive changes on non-substituted $\mathrm{CuPc}$-covered electrodes and exploited such effect in sensing devices [52]. From Fig. 6, one can observe also that once the light is turned off the current decreases and recovers its initial in-dark drift. At higher moisture content (RH $\sim 40-45 \%)$ the ionic conductivity prevails in the sample and charge storage is not effective as for measurements taken in RH $10 \%$. Based on the work of Nam et al. to explain the photocurrent response of heme proteinbased diodes $[58,59]$, we believe the same model can be taken here to explain the electrical response of CuTsPcprinted films on paper, shown in Fig. 6. For Nam et al. the protein heme group acts as a semiconductor that absorbs light and produces excitons, whereas the protein amino acid surroundings are intrinsically insulating and represent a potential barrier for the photogenerated charges. Here, the semiconducting CuTsPc molecules are embedded into paper and photogenerated electron-hole pairs (excitons) are confined by the paper insulating fibers. However, the insulating characteristics of paper fibers is strongly affected by $\mathrm{RH}$, thus depending the humidity levels the extraction photogenerated charges can be more or less effective.
Therefore, to exploit the semiconducting properties of CuTsPc on paper-based electronics one has to consider the strong influence of humidity on the device electronic and optoelectronic properties.

\section{Conclusions}

In the present work, we demonstrate the feasibility of the thermal inkjet printing of $\mathrm{CuTsPc}$ films onto paper substrates and the effects related to humidity on the sample electrical properties. DC measurements revealed that sample resistance decreases sharply from $10^{12} \Omega$ in moderate vacuum to approximately $10^{7} \Omega$ at highly water-saturated atmosphere. The sample electrical response was attributed to electronic charge carriers from the CuTsPc molecules and ionic species such as paper impurities and $\mathrm{Na}^{+}$ions dissociated from CuTsPc molecule. The strong dependence of the sample resistance on RH levels is related mainly to its ionic conduction. Additionally, resistance values decrease as the number of prints increase from 1-5, followed by a constant behavior as the number of prints further rises. This effect might be related to the ink penetration into the paper that reaches a limit on its absorptivity. The effects of humidity on the electrical properties of $\mathrm{CuTsPc}$ were also investigated as a function of temperature. From this analysis, we observed the semiconducting properties of CuTsPc arise above approximately $70{ }^{\circ} \mathrm{C}$ that corresponds to water release (11\% of mass) from the sample, as corroborated by TGA/ DTG and DSC measurements. Upon white light illumination, the semiconducting properties of CuTsPc molecules can be also observed regardless of humidity, whereas the charge carriers extraction is governed by the RH level. The results presented here show that electronic and optoelectronic applications exploiting the semiconducting properties of CuTsPc in paper electronics must take into account effects from the presence of humidity.

Acknowledgements The authors thank FAPESP and INEO (Brazil) for the financial support.

\section{References}

1. Kao P-C, Chu S-Y, You Z-X et al (2006) Improved efficiency of organic light-emitting diodes using CoPc buffer layer. Thin Solid Films 498:249-253

2. Hohnholz D, Steinbrecher S, Hanack M (2000) Applications of phthalocyanines in organic light emitting devices. J Mol Struct 521:231-237

3. Pakhomov GL, Pakhomov LG, Travkin VV et al (2010) Phthalocyanine-based photoelectrical cells: effect of environment on power conversion efficiency. J Mater Sci 45:1854-1858

4. Vasseur K, Rand BP, Cheyns D et al (2011) Structural evolution of evaporated lead phthalocyanine thin films for near-infrared sensitive solar cells. Chem Mater 23:886-895 
5. Tai Y, Guo Z-C, Sharma J (2011) Improved charge separation properties of organic hetero-junction solar cells by self-assembled monolayers anchored Ag nanoparticles. J Nanosci Nanotechnol 11:10813-10816

6. Luo X, Xu L, Xu B, Li F (2011) Electrodeposition of zinc oxide/ tetrasulfonated copper phthalocyanine hybrid thin film for dyesensitized solar cell application. Appl Surf Sci 257:6908-6911

7. Boeckler C, Feldhoff A, Oekermann T (2007) Electrodeposited zinc oxide/phthalocyanine films - an inorganic/organic hybrid system with highly variable composition. Adv Funct Mater 17:3864-3869

8. Bao Z, Lovinger AJ, Dodabalapur A (1996) Organic field-effect transistors with high mobility based on copper phthalocyanine. Appl Phys Lett 69:3066-3068

9. Tang Q, Li H, He M et al (2006) Low threshold voltage transistors based on individual single-crystalline submicrometersized ribbons of copper phthalocyanine. Adv Mater 18:65-68

10. Zeis R, Siegrist T, Kloc C (2005) Single-crystal field-effect transistors based on copper phthalocyanine. Appl Phys Lett 86:022103

11. Alessio P, de Oliveira RF, Aoki PHB et al (2012) Molecular architecture and electrical properties in evaporated films of cobalt phthalocyanine. J Nanosci Nanotechnol 12:7010-7020

12. Zeyada HM, El-Nahass MM (2008) Electrical properties and dielectric relaxation of thermally evaporated zinc phthalocyanine thin films. Appl Surf Sci 254:1852-1858

13. Ghani F, Kristen J, Riegler H (2012) Solubility properties of unsubstituted metal phthalocyanines in different types of solvents. J Chem Eng Data 57:439-449

14. Zanfolim AA, Volpati D, Olivati CA et al (2010) Structural and electric-optical properties of zinc phthalocyanine evaporated thin films: temperature and thickness effects. J Phys Chem C 114:12290-12299

15. Berggren M, Nilsson D, Robinson ND (2007) Organic materials for printed electronics. Nat Mater 6:3-5

16. Logothetidis S (2008) Flexible organic electronic devices: materials, process and applications. Mater Sci Eng B 152:96-104

17. Teichler A, Perelaer J, Schubert US (2013) Inkjet printing of organic electronics - comparison of deposition techniques and state-of-the-art developments. J Mater Chem C 1:1910-1925

18. Locklin J, Shinbo K, Onishi K et al (2003) Ambipolar organic thin film transistor-like behavior of cationic and anionic phthalocyanines fabricated using layer-by-layer deposition from aqueous solution. Chem Mater 15:1404-1412

19. Benten H, Kudo N, Ohkita H, Ito S (2009) Layer-by-layer deposition films of copper phthalocyanine derivative; their photoelectrochemical properties and application to solution-processed thinfilm organic solar cells. Thin Solid Films 517:2016-2022

20. Fernandes EGR, Vieira NCS, de Queiroz AAA et al (2010) Immobilization of poly(propylene imine) dendrimer/nickel phtalocyanine as nanostructured multilayer films to be used as gate membranes for SEGFET pH sensors. J Phys Chem C 114:64786483

21. Chaidogiannos G, Petraki F, Glezos N et al (2008) Soluble substituted phthalocyanines for OFET applications. Mater Sci Eng B 152:105-108

22. Lian K, Li R, Wang $\mathrm{H}$ et al (2010) Printed flexible memory devices using copper phthalocyanine. Mater Sci Eng B 167: $12-16$

23. Kim Y, Do KimJP, Kwon OS, Cho IH (2009) The synthesis and application of thermally stable dyes for ink-jet printed LCD color filters. Dye Pigment 81:45-52

24. Cui L, Pu T, Liu Y, He X (2013) Layer-by-layer construction of graphene/cobalt phthalocyanine composite film on activated GCE for application as a nitrite sensor. Electrochim Acta 88:559-564
25. Zucolotto V, Ferreira M, Cordeiro MR et al (2006) Nanoscale processing of polyaniline and phthalocyanines for sensing applications. Sensors Actuators B Chem 113:809-815

26. Volpati D, Alessio P, Zanfolim AA et al (2008) Exploiting distinct molecular architectures of ultrathin films made with iron phthalocyanine for sensing. J Phys Chem B 112:15275-15282

27. Šebera J, Nešpůrek S, Kratochvílová I et al (2009) Charge carrier mobility in sulphonated and non-sulphonated $\mathrm{Ni}$ phthalocyanines: experiment and quantum chemical calculations. Eur Phys J B 72:385-395

28. Rolland JP, Mourey DA (2013) Paper as a novel material platform for devices. MRS Bull 38:299-305

29. Tobjörk D, Österbacka R (2011) Paper electronics. Adv Mater 23:1935-1961

30. Zheng G, Cui Y, Karabulut E et al (2013) Nanostructured paper for flexible energy and electronic devices. MRS Bull 38:320-325

31. Hu L, Choi JW, Yang Y et al (2009) Highly conductive paper for energy-storage devices. Proc Natl Acad Sci USA 106:21490-21494

32. Hu L, Wu H, Cui Y (2010) Printed energy storage devices by integration of electrodes and separators into single sheets of paper. Appl Phys Lett 96:183502

33. Nyström G, Razaq A, Strømme M et al (2009) Ultrafast allpolymer paper-based batteries. Nano Lett 9:3635-3639

34. Zschieschang U, Yamamoto T, Takimiya K et al (2011) Organic electronics on banknotes. Adv Mater 23:654-658

35. Kim Y-H, Moon D-G, Han J-I (2004) Organic TFT array on a paper substrate. IEEE Electron Device Lett 25:702-704

36. Bollström R, Määttänen A, Tobjörk D et al (2009) A multilayer coated fiber-based substrate suitable for printed functionality. Org Electron 10:1020-1023

37. Mannerbro R, Ranlöf M, Robinson N, Forchheimer R (2008) Inkjet printed electrochemical organic electronics. Synth Met 158:556-560

38. Yun S, Jang S-D, Yun G-Y et al (2009) Paper transistor made with covalently bonded multiwalled carbon nanotube and cellulose. Appl Phys Lett 95:104102

39. Lamprecht B, Thünauer R, Ostermann M et al (2005) Organic photodiodes on newspaper. Phys status solidi 202:R50-R52

40. Wang F, Chen Z, Xiao L et al (2010) Papery solar cells based on dielectric/metal hybrid transparent cathode. Sol Energy Mater Sol Cell 94:1270-1274

41. Gomes TC, Constantino CJL, Lopes EM et al (2012) Thermal inkjet printing of polyaniline on paper. Thin Solid Films 520:7200-7204

42. Derby B (2010) Inkjet printing of functional and structural materials: fluid property requirements, feature stability, and resolution. Annu Rev Mater Res 40:395-414

43. Murphy EJ (1960) The dependence of the conductivity of cellulose, silk and wool on their water content. J Phys Chem Solids 16:115-122

44. Martin M, André J-J, Simon J (1983) Influence of dioxygen on the junction properties of metallophthalocyanine based devices. J Appl Phys 54:2792-2794

45. Pankow JW, Arbour C, Dodelet JP et al (1993) Photoconductivity/dark conductivity studies of chlorogallium phthalocyanine thin films on interdigitated microcircuit arrays. J Phys Chem 97:8485-8494

46. Bohrer FI, Colesniuc CN, Park J et al (2009) Comparative gas sensing in cobalt, nickel, copper, zinc, and metal-free phthalocyanine chemiresistors. J Am Chem Soc 131:478-485

47. Passard M, Maleysson C, Pauly A et al (1994) Gas sensitivity of phthalocyanine thin films. Sensors Actuators B Chem 19:489-492

48. Schöllhorn B, Germain JP, Pauly A et al (1998) Influence of peripheral electron-withdrawing substituents on the conductivity 
of zinc phthalocyanine in the presence of gases. Part 1: reducing gases. Thin Solid Films 326:245-250

49. Germain JP, Pauly A, Maleysson C et al (1998) Influence of peripheral electron-withdrawing substituents on the conductivity of zinc phthalocyanine in the presence of gases. Part 2: oxidizing gases. Thin Solid Films 333:235-239

50. Ho K-C, Tsou Y-H (2001) Chemiresistor-type NO gas sensor based on nickel phthalocyanine thin films. Sensors Actuators B Chem 77:253-259

51. Bohrer FI, Sharoni A, Colesniuc C et al (2007) Gas sensing mechanism in chemiresistive cobalt and metal-free phthalocyanine thin films. J Am Chem Soc 129:5640-5646

52. Karimov KS, Qazi I, Khan TA et al (2008) Humidity and illumination organic semiconductor copper phthalocyanine sensor for environmental monitoring. Environ Monit Assess 141:323328

53. Jarota A, Brozek-Pluska B, Czajkowski W, Abramczyk H (2011) Water confined in films of sulphonated phthalocyanines. J Phys Chem C 115:24920-24930

54. Harbeck S, Atilla D, Dülger I et al (2014) The role of hydrogen bonding in the sensitivity of QCM sensors: a spectroscopic study on tosylamido phthalocyanines. Sensors Actuators B Chem 191:750-756

55. Harbeck S, Emirik ÖF, Gürol I et al (2013) Understanding the VOC sorption processes on fluoro alkyl substituted phthalocyanines using ATR FT-IR spectroscopy and QCM measurements. Sensors Actuators B Chem 176:838-849

56. Barbosa C, Ferreira A (2002) Preparation and characterization of $\mathrm{Cu}$ (II) phthalocyanine tetrasulfonate intercalated and supported on layered double hydroxides. J Incl Phenom Macrocycl Chem 42:15-23

57. Yamamoto K, Egusa S, Sugiuchi M, Miura A (1993) Photogeneration mechanism of charged carriers in copper-phthalocyanine thin films. Solid State Commun 85:5-10

58. Nam S, Kim H, Degenaar P et al (2012) Extremely slow photocurrent response from hemoprotein films in planar diode geometry. Appl Phys Lett 101:223701

59. Nam S, Kim H, Kim Y (2010) Bias-dependent photocurrent response in protein nanolayer-embedded solid state planar diode devices. Nanoscale 2:694-696 